\title{
Hybrid electro-optics and chipscale integration of electronics and photonics
}

L. Dalton, B. Robinson, D. Elder, A. Tillack, L. Johnson

L. R. Dalton, B. H. Robinson, D. L. Elder, A. F. Tillack, L. E. Johnson, "Hybrid electro-optics and chipscale integration of electronics and photonics," Proc. SPIE 10364, Organic Sensors and Bioelectronics X, 1036402 (25 August 2017); doi: 10.1117/12.2278795

Event: SPIE Organic Photonics + Electronics, 2017, San Diego, California, United States 


\title{
Hybrid Electro-Optics and Chipscale Integration of Electronics and Photonics
}

\author{
L. R. Dalton*, B. H. Robinson, D. L. Elder, A. F. Tillack ${ }^{\#}$, and L. E. Johnson \\ Department of Chemistry, University of Washington, Seattle, WA 98195-1700 \\ ${ }^{\#}$ Current address: Center for Computational Sciences, Oak Ridge National Laboratory, One \\ Bethel Valley Road, P.O. Box 2008, MS-6008, Oak Ridge, TN 37831
}

\begin{abstract}
Taken together, theory-guided nano-engineering of organic electro-optic materials and hybrid device architectures have permitted dramatic improvement of the performance of electro-optic devices. For example, the voltage-length product has been improved by nearly a factor of $10^{4}$, bandwidths have been extended to nearly $200 \mathrm{GHz}$, device footprints reduced to less than $200 \mu \mathrm{m}^{2}$, and femtojoule energy efficiency achieved. This presentation discusses the utilization of new coarse-grained theoretical methods and advanced quantum mechanical methods to quantitatively simulate the physical properties of new classes of organic electro-optic materials and to evaluate their performance in nanoscopic device architectures, accounting for the effect on chromophore ordering at interfaces in nanoscopic waveguides.
\end{abstract}

Keywords: Organic electro-optic materials, multiscale theoretical calculations, interfaces, 2-D materials, plasmonics, silicon photonics

\section{INTRODUCTION}

Chipscale integration of electronics and photonics is well recognized as a critical next step in the evolution of information technology and the electro-optic modulator ${ }^{1}$ is a key element in this integration. However, the achievement of this objective is a formidable challenge due to the current substantial size mismatch of electronic and photonic circuit elements, together with drive voltage requirements, energy efficiency, optical insertion loss, and bandwidth of the active optical components. The advent of silicon photonics ${ }^{2-6}$ and plasmonics ${ }^{7-9}$ has provided hope for overcoming these limitations and ultimately permitting chipscale integration of electronics and photonics. A critical parameter for assessing the potential of realizing chipscale integration is the voltage length parameter, $U_{\pi} L$ of electro-optic modulators ${ }^{10}$. Device footprint, insertion loss and bandwidth improve with decreasing device length; however, drive voltage requirements increase with decreasing device length, which also leads to poorer energy efficiency. An obvious way to improve (decrease) $U_{\pi} L$ is to increase electrooptic activity. Another way to improve $U_{\pi} L$ is through improved device architecture, e.g., slotted silicon photonic modulators or plasmonic modulators. Both of these architectures concentrate electrical and optical fields in nanoscopic dimensions and the plasmonic modulator has the added advantage of "slow light" enhancement.

${ }^{\#}$ This manuscript has been authored by UT-Battelle, LLC under Contract No. DE-AC05-00OR22725 with the U.S. Department of Energy. The United States Government retains and the publisher, by accepting the article for publication, acknowledges that the United States Government retains a non-exclusive, paid-up, irrevocable, worldwide license to publish or reproduce the published form of this manuscript, or allow others to do so, for United States Government purposes. The Department of Energy will provide public access to these results of federally sponsored research in accordance with the DOE Public Access Plan (http://energy.gov/downloads/doepublic-access-plan). 
In this communication, we focus on improving the electro-optic activity of organic materials through theoryguided design ${ }^{11-17}$ of chromophores and their incorporation into matrices of controlled orientational dimensionality ${ }^{11,14}$. Use of multiscale theoretical methods is critical because many intermolecular interactions compete to determine electric field poling induced electro-optic activity of organic materials and simple trial and error synthesis of new chromophores is too time consuming and costly.

It is generally assumed that poling-induced electro-optic activity will be independent of device architecture (interactions at material/device electrode interfaces); however, it has recently observed that electro-optic activity depends on the width of slotted silicon-organic hybrid $(\mathrm{SOH})$ waveguide modulators and plasmonic-organic hybrid waveguide modulators $(\mathrm{POH})^{10}$. Multiscale theoretical methods are necessary to simulate the influence of interactions at interfaces ${ }^{10}$

Such calculations permit understanding chromophore organization both in the bulk organic electro-optic (OEO) material and at the interface of the OEO material with the electrode. The effect of interfacial interactions is evident in devices with slot widths of up to $100 \mathrm{~nm}$; beyond that length, bulk interactions dominate. ${ }^{10}$

A number of improvements in coarse-grained statistical mechanical computational methods have been required to successfully model the overall morphology of organic electro-optic materials under electric field poling. The $\pi$-electron segments of OEO materials are most effectively modeled by ellipsoids rather than commonly used spheres. The number of ellipsoids needed to model a chromophore depends on the details of chromophore structure; this is referred to as the Level of Detail (LoD) method ${ }^{13}$. For example, two ellipsoids are required to effectively represent chromophore curvature. Use of multiple ellipsoids can improve the quality of the simulation but comes at the price of computation time so the goal is to use the lowest LoD that leads to both effective simulation and the fastest simulation. Another modification addresses the problem of computational trajectories hanging up in non-equilibrium distributions. In this approach, referred to as the Adiabatic Volume Adjustment (AVA) method ${ }^{13}$, the simulation mimics the condensation of chromophores from the gas phase or from solution. That is, the calculation is started at a reduced chromophore number density and the number density is smoothly increased to near the actual equilibrium number density. The final equilibrium density is obtained using the NPT ensemble during equilibrium simulations.

New and improved coarse-grained Monte Carlo simulations have motivated the transition from low number density chromophore-polymer composite materials to high number density neat chromophore materials ${ }^{11,13-17}$, to materials of reduced orientation dimensionality by utilization of non-chromophore dipolar and quadrupolar interactions ${ }^{11,14}$, and to binary chromophore materials ${ }^{11}$. These calculations have also proven crucial for the simulation of electro-optic activity in slotted silicon photonic and plasmonic waveguides with widths less than $100 \mathrm{~nm}^{10}$. Thus, such simulations have permitted understanding of the importance of various intermolecular interactions in bulk materials and of chromophore-electrode interactions at interfaces. It is also important to utilize chromophore distributions from coarse-grained statistical mechanical calculations in quantum mechanical calculations of linear and nonlinear optical properties. Use of such distributions permit meaningful evaluation of excitonic contributions to linear and nonlinear optical properties.

Electro-optic activity (e.g., the principal element, $\mathrm{r}_{33}$, of the electro-optic tensor for electrically poled OEO materials) is linearly proportional to the product of three quantities: 1 ) the molecular first hyperpolarizability $\left.\left(\beta_{z z z}\right) ; 2\right)$ the chromophore number density $\left(\rho_{\mathrm{N}}\right)$; and 3$)$ the acentric order parameter $\left(<\cos ^{3} \theta>\right.$ where $\theta$ is the angle between the applied electric field (electrical and optical fields assumed to be co-linear) and the EO chromophore principal tensor axis. These three quantities are typically assumed to be independent. However, as will be discussed herein, they are in fact highly interdependent. Coarse-grained statistical mechanical simulations can be employed to compute the product $\rho_{N}<\cos ^{3} \theta>$ and it is this product that must be considered in optimizing OEO activity. Chromophore modification to improve acentric order will also affect chromophore number density so that optimization cannot be achieved by simply focusing on acentric order. Chromophore organization (and thus acentric order) will impact $\beta_{z z z}$ through chromophore-chromophore excitonic interactions in the most extreme case or through impact on the dielectric environment in the case of weaker intermolecular 
interactions. Because of this interdependence of the above three parameters, careful consideration must be given to the impact of chromophore modification and material processing (spin casting, poling, and lattice hardening subsequent to poling) on the product $\beta_{z z z} \rho_{\mathrm{N}}<\cos ^{3} \theta>$ and not just on individual parameters. In an analogous manner, the critical device parameter $U_{\pi} L$ for a silicon-organic hybrid modulator is commonly related to electrooptic activity by the expression: $U_{\pi} L=\lambda w / n^{3} r_{33} \Gamma$, where $\lambda$ is the vacuum wavelength of the optical carrier, $w$ is the electrode spacing (slot width), $n$ is the index of refraction, and $\Gamma$ is the field interaction factor, which is a measure of how strongly the applied fields interact (overlap) with the OEO material in the slot. For a plasmonicorganic hybrid modulator, the expression becomes $U_{\pi} L=\lambda w / n^{3} r_{33} \Gamma_{E} n_{\text {slow }}$ where $\Gamma_{\mathrm{E}}$ denotes the field energy interaction factor and $n_{\text {slow }}$ reflects the effect of reduced energy velocity in plasmonic slot waveguides. Again, the parameters are typically assumed to be independent but here we will demonstrate that $\mathrm{r}_{33}$ depends on $w$ for the narrowest waveguides. From the above expressions it is seen that $U_{\pi} L$ is predicted to improve (decrease) as $w$ is decreased assuming that $\mathrm{r}_{33}$ does not change with changing $w$. Now we will consider how $\mathrm{r}_{33}$ changes with $w$ for widths smaller than $100 \mathrm{~nm}$ (presently, limiting device performance) and how this deficiency can be overcome. At present, the best $U_{\pi} L$ values are on the order of $40 \mathrm{~V} \mu \mathrm{m}$ (for POH devices) ${ }^{10}$. Here we focus on how this value can be improved to 4-10 V $\mu \mathrm{m}$ through chromophore modification.

Chromophore modification must serve several purposes. In addition to the optimization of the product of chromophore number density and acentric order, chromophore modification must permit lattice hardening (chromophore crosslinking) to glass transition temperatures on the order of $200^{\circ} \mathrm{C}$ (to realized desired thermal and photochemical stability in device operation) and processability appropriate for spin casting and electric field poling. Each of these desired features must be considered in the design of the chromophores.

\section{THEORY}

Computational modeling of poled organic electro-optic systems is an inherently multi-scale problem ${ }^{10,13,16}$ due to their amorphous but statistically ordered structure and complex interactions with light. However, simulations for understanding EO systems can be partitioned initially into two primary domains (1) electronic structure modeling to study optical properties, and (2) classical condensed-phase simulations for studying structural properties, with substantial exchange between the two domains: generation of force field parameters (moving from $\mathrm{QM}$ to classical) or representative configurations (moving from classical to $\mathrm{QM}$ ).

Electronic structure calculations on EO systems are typically performed with density functional theory (DFT) due to system size ( $\sim 1000$ basis functions/molecule), though higher-level methods are feasible on modern HPC systems. However, common DFT methods containing small quantities of Hartree-Fock (HF) exchange, such as B3LYP, are not able to accurately distinguish the relative hyperpolarizabilities of high-performance chromophores $^{12,18-20}$ such as EZ-FTC, CLD-1, YLD-156, and YLD-124, even after correcting for solvent and resonance effects. The structures of these chromophores and their relative hyperpolarizabilities are shown in Figure 1.

Accurately representing the charge distribution of high-performance chromophores, such as the four shown above and their derivatives, requires careful treatment of exact (HF) exchange in the calculation protocol. Functionals containing $>50 \%$ long-range HF exchange have been found to yield reasonable relative predictions of hyperpolarizabilities when compared to known standards ${ }^{12,18,19}$ However, exact hyperpolarizabilty and charge transfer excitation energies vary almost linearly with the amount of long-range HF exchange ${ }^{12,19}$ indicating that results should not be compared between functionals, and that DFT-calculated absolute hyperpolarizability values are of limited utility. Figure 2(a) illustrates this phenomenon using a series of calculations on the common chromophore CLD-1 using different DFT functionals ${ }^{12}$

DFT calculations using high-HF DFT functionals can also be used to explore the effect of the dielectric environment surrounding chromophores, which is crucial due to the high dielectric constant of EO films. ${ }^{18}$ Figure 2(b) shows a set of calculations on CLD-1 and YLD-156 in different solvents compared with 
experimental hyper-Rayleigh scattering (HRS) results from Bale et al. ${ }^{18}$; unlike in the prior work, differences in the calculated results between the two chromophores are distinguishable and consistent with experiment. The dielectric dependence also increases at different rates depending on the chromophore structure.

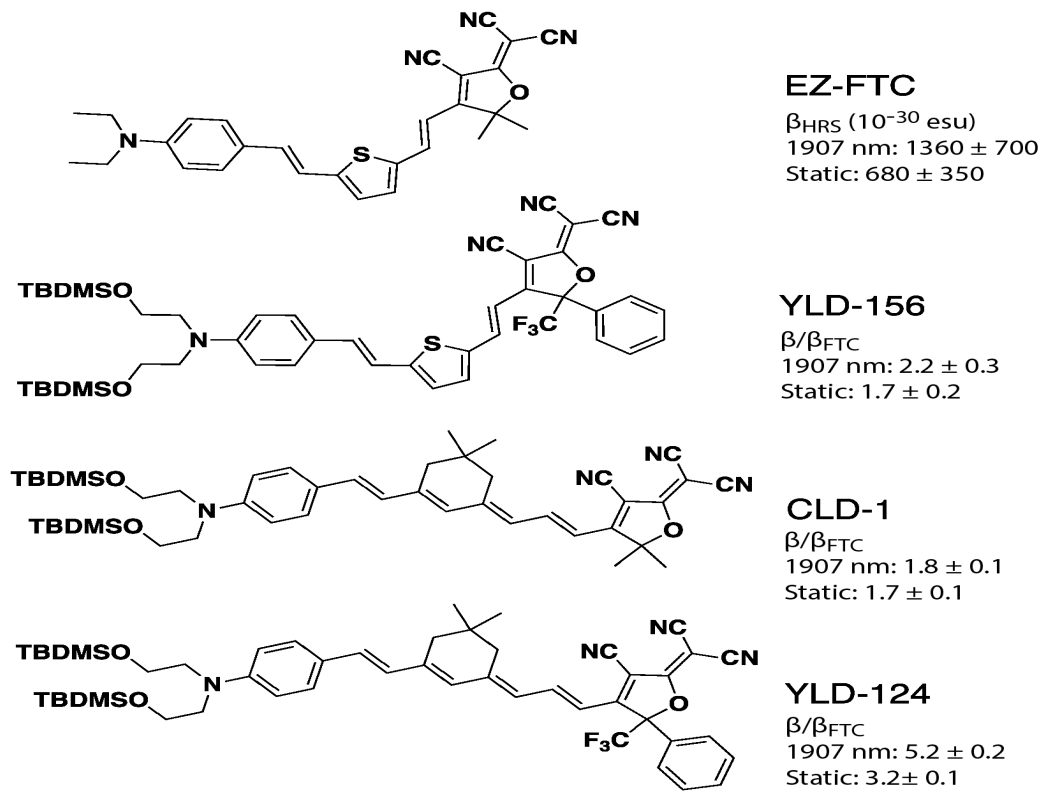

Figure 1. Hyperpolarizabilities of high-performance chromophores EZ-FTC, CLD-1, YLD-156, and YLD-124 in chloroform from hyperRayleigh scattering experiments are shown. ${ }^{18}$ Relative static hyperpolarizabilities were extrapolated using a damped two-level model with a linewidth parameter of $0.1 \mathrm{eV} .^{18}$

Thus, advances in DFT functional development and calculation protocol design have allowed for experimentally-relevant accuracy in evaluating the relative hyperpolarizabilities of chromophores, including consistency with experimentally predicted advantages for state-of-the-art chromophores such as YLD-124 and its derivatives versus YLD-156 and CLD-1). ${ }^{18}$ These protocols can now be used as a design tool for development of new chromophores, instead of primarily for post-hoc explanation of experimental data.

As mentioned above, the electro-optic coefficient, $r_{33}$ depends on the product of the hyperpolarizability and the loading parameter. The loading parameter is the product of the chromophore number density $\left(\rho_{\mathrm{N}}\right)$ and the acentric order parameter $\left\langle\cos ^{3} \theta\right\rangle$. Here we consider the simulation of the electro-optic coefficient, in which we have estimated the Pockels' hyperpolarizability of YLD-124 to be $8100 \cdot 10^{-30}$ esu and simulate the density and order using coarse-grained Monte Carlo simulations, representing YLD-124 using 19 ellipsoids fit based on a DFT-derived charge distribution and Lennard-Jones parameters from the OPLSAA all-atom force field. Figure 3 shows the simulated EO coefficient as a function of the external electric field.

The chromophore core of YLD-124 is defined by two ellipsoids that are bent by about $15^{\circ}$ relative to each other. The other ellipsoids are needed to mimic the effects of the pendant groups around the core. The complete representation is shown in the inset in Figure 3. In particular, the protecting groups on the nitrogen donor require 14 ellipsoids to simulate due to their large number of conformational degrees of freedom compared to the rigid conjugated systems in the core and in the acceptor. To explore the effects of changing the size of the protecting groups on the donor and other locations, we considered a further coarse-grained model of this type of chromophore, in which the various protecting groups are replaced with one or two protecting discs centered about the center of each of the two core ellipsoids, approximating averaging over many side chain configurations. The effect of the size of the protecting groups is demonstrated by keeping the core ellipsoids and 
those on the acceptor fixed, and varying only the size of a single ellipsoid on the donor side, and optimally protecting the dipole in the ellipsoid near the donor.

a)

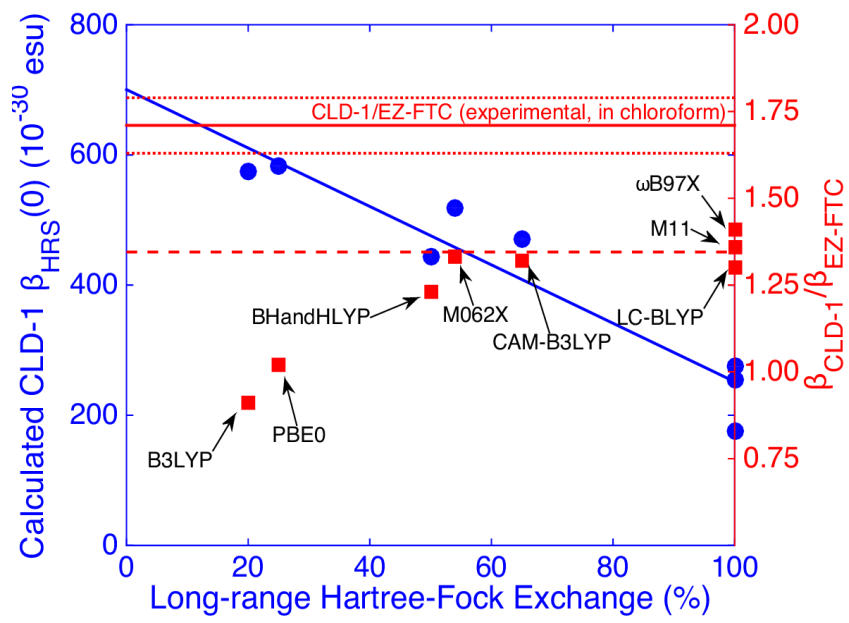

b)

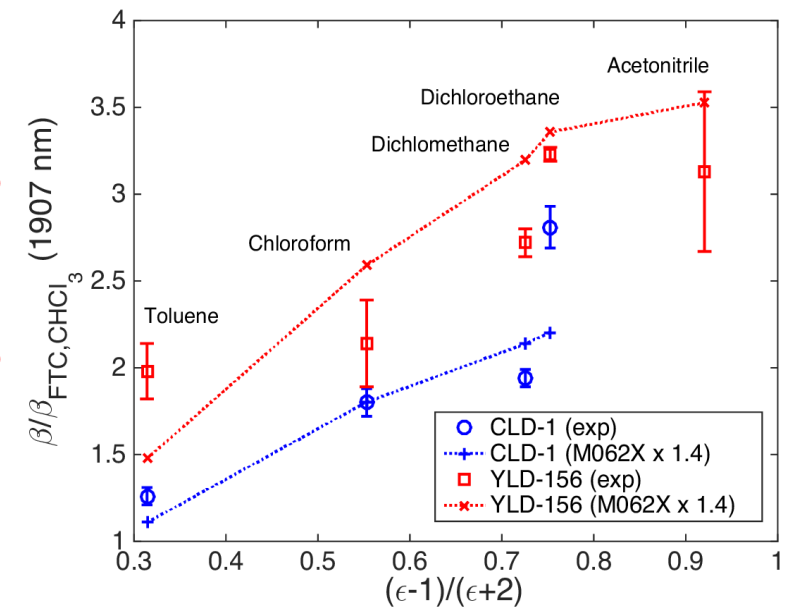

Figure 2. a) Calculations on CLD-1 are shown for eight different DFT functionals using the 6-31+G(d) basis set in PCM chloroform using Gaussian $09^{40}$. While the absolute static hyperpolarizability (blue circles) decreases linearly (squared correlation coefficient $\mathrm{R}^{2}=0.90$ ) with increasing long-range HF exchange, the ratio of the hyperpolarizability of CLD-1 and benchmark chromophore EZ-FTC (red squares) becomes stable above 50\% long-range HF exchange (dashed red line), though it remains lower than the experimental value (solid red line) ${ }^{12}$. b) Dielectric enhancement of the relative hyperpolarizability of CLD-1 and YLD-156 chromophores calculated at the M062X/6-31+G(d) level of theory in the presence of a IEF-PCM reaction field using Gaussian $09^{21}$, and compared with experimental results, and estimates of error, from Bale et al. ${ }^{18}$ Computational results were scaled by a constant factor of 1.4 and frequency dependence was introduced by applying a damped two-level model using the experimental wavelength of $1907 \mathrm{~nm}$ and a linewidth of $0.1 \mathrm{eV}$.

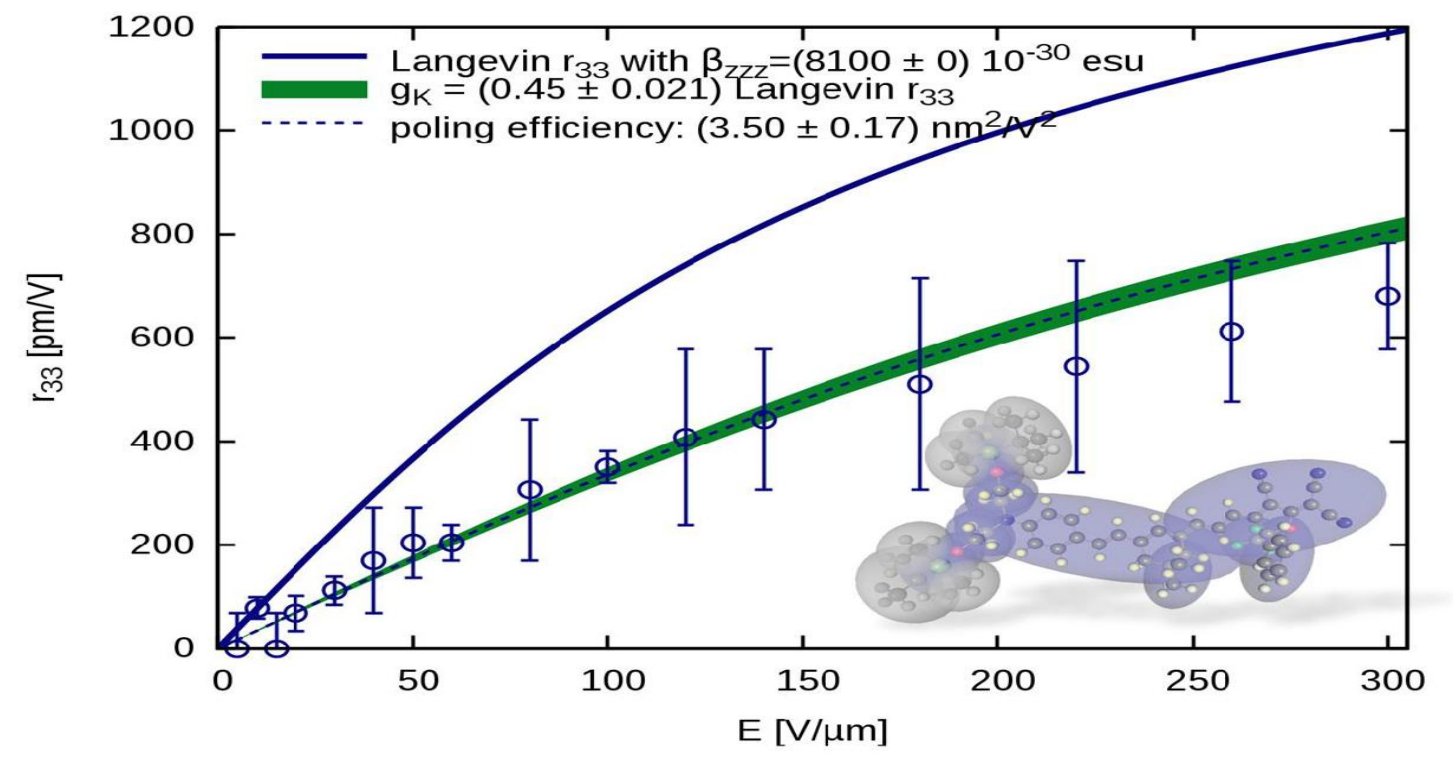

Figure 3: Simulated electro-optic coefficient, $r_{33}$, as a function of poling field strength. Blue circles show ensemble averages for individual simulations at different poling strengths, assuming $\beta_{z z z}(-\omega ; \omega, 0)=8100 \cdot 10^{-31} \mathrm{esu}$, for an NPT ensemble, at one atmosphere and $420 \mathrm{~K}$, using AVA to prepare the system, for 80,000 Monte Carlo cycles, consisting of 108 chromophores of racemic, neat YLD124 represented by 19 ellipsoids in each chromophore [Tillack, A. F., Electro-optic material design criteria derived from condensed matter simulations using the Level-of-Detail coarse-graining approach. Ph.D. Thesis, University of Washington, Seattle (2015)]. The individual simulations are compared with the $r_{33}$ using the simulated density and the $\left\langle\cos ^{3} \theta\right\rangle$ predicted by Langevin (or independent particle) theory. The green line is the Langevin limit attenuated by the average computed Kirkwood factor, $g_{K}$, which represents the degree of cooperativity for dipole 
orientation (in the Langevin limit, $g_{K}=1$ ). The poling efficiency, $\frac{r_{33}}{E}$, of $3.5\left(\frac{\mathrm{nm}}{\mathrm{V}}\right)^{2}$ is the limiting efficiency (as the slope of the green line) at zero field.

In this example, the loading remains large for protecting group sizes, up to about $5 \AA$ and is comparable to the value of the full ellipsoid representation of YLD124 (shown in Figure 3, above), which has a 0.85 loading. An improvement of about $50 \%$ in the acentric order can be achieved at the optimum radius, $\sim 3.5 \AA$. The drop off of the loading parameter at larger radii is due to the combination of larger molecular size (diluting the concentration of chromophores) and a modest reduction in overall chromophore order. Similar results were found when two protecting groups were considered. ${ }^{10}$

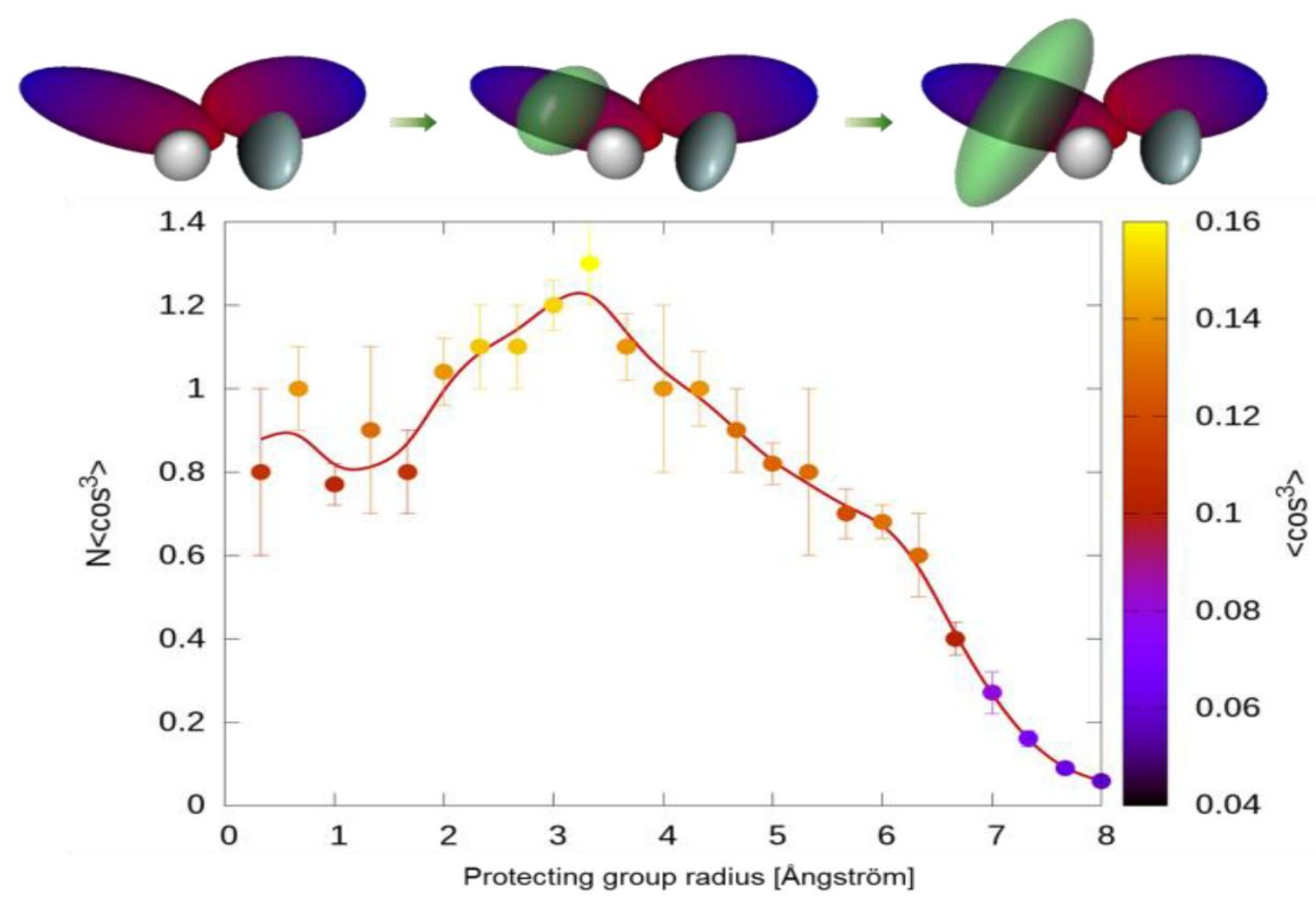

Figure 4: Chromophore loading, $N\left\langle\cos ^{3} \theta\right\rangle$, where $N=\varrho\left[10^{20}\right.$ molecules $\left./ c c\right]$, is shown as a function of the protecting group radius for a protecting group on the center of the donor portion of the chromophore core. Simulations follow the same protocol described in Figure 3. The loading parameter for YLD124 (from Figure 3) is 0.85. [Tillack, A. F., Electro-optic material design criteria derived from condensed matter simulations using the Level-of-Detail coarse-graining approach. Ph.D. Thesis, University of Washington, Seattle, (2015)].

Given that moderate improvement in the loading parameter can be achieved through the addition of various pendant groups, we now consider changing the shape of the core. Figure 5 shows how the protecting groups alter the loading parameter when the core has the two ellipsoids collinear, rather than bent (as shown in Figures 3 and 4). Other than the relative orientation of the two ellipsoids that comprise the chromophore core, all other parameters of the core ellipsoids are kept constant. Aligning the donor and acceptor also makes the chromophore more like an ideal dipole, increasing the torque that a poling field can exert on the molecule so long as adequate dipole protection is provided to prevent centrosymmetric pairing. It is important to realize that the change in core geometry from bent to linear provides one with an opportunity to increase the loading parameter by two-fold from that seen for YLD124, though the linear geometry is also more sensitive to dipole protection, requiring protecting groups of $>3 \AA$ before gaining an advantage over the bent geometry. However, there is a limit to which one can add protecting groups beyond which the loss of chromophore density lowers the 
loading parameter to unacceptable levels even if substantial acentric order is retained. Therefore, while protecting groups of modest size can be beneficial, one does not want to add large, bulky pendant groups (larger than the optimal size for the core geometry of the chromophore) unless they perform a crucial function such as providing moieties for crosslinking or influencing the glass transition temperature of the material in a desired manner. Large protecting groups do (continue to) improve order as the radius increases, but at the expense of chromophore density (and hence EO performance).

Both the straight and bent cores give an optimal loading with a modest sized protecting group, of around $3.5-4.0 \AA$ radius. In particular the linear core has an effective loading parameter that is about twice what was obtained from the simulation of YLD124.

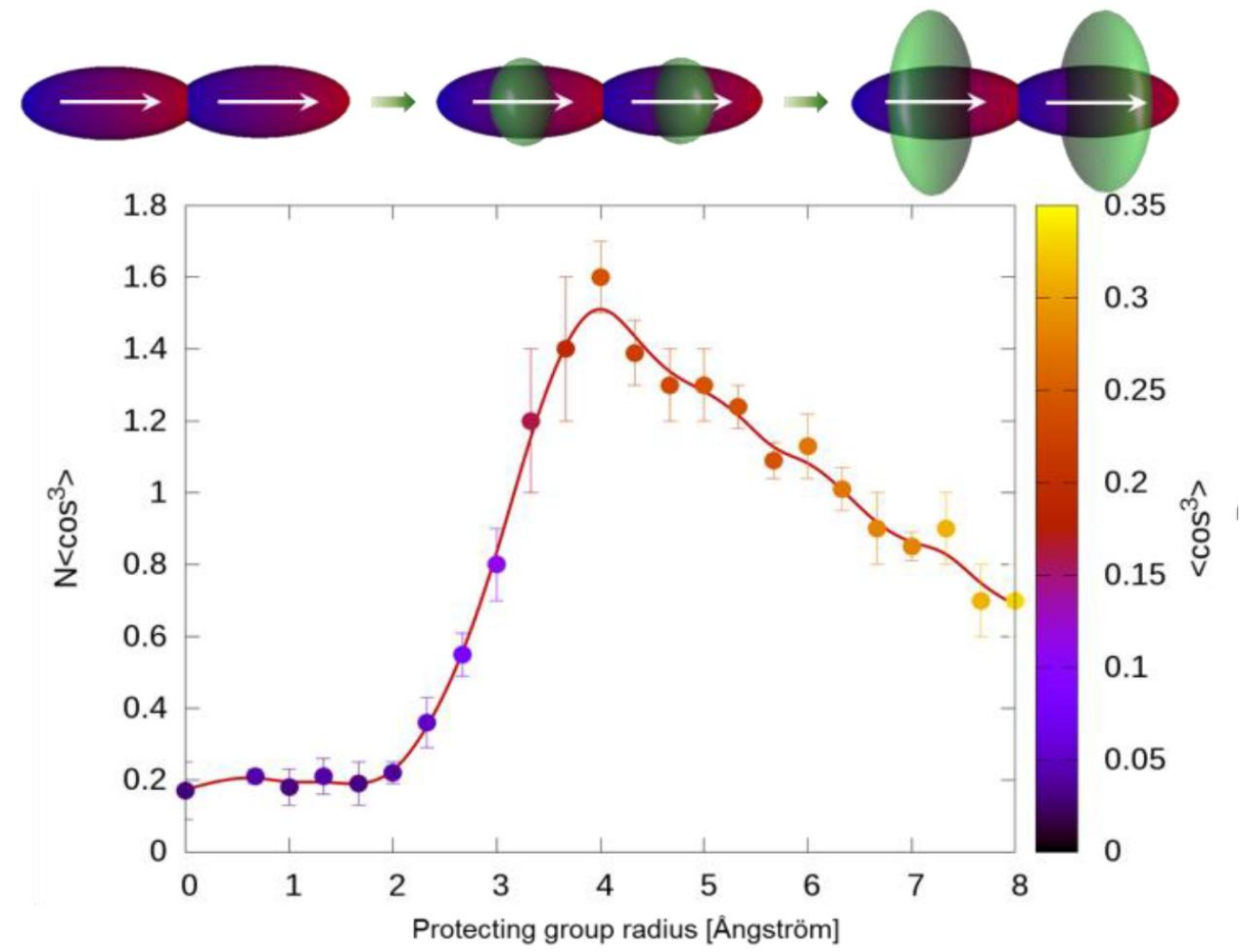

Figure 5. Chromophore loading is shown as a function of protecting group radii for a hypothetical YLD-124-like chromophore with a straight core. Simulation protocol was the same as described above. ${ }^{10}$ The structure of the molecule, at the peak in loading, is shown at the top. The green is drawn to scale, and appears to not be very large to be effective: This is incorrect, the protecting group is a disc, and when seen sideways it does extend well above the surface of the core ellipsoids. The disc radius is over $4 \AA$, and the ellipsoid in the $\mathrm{z}$ dimension is about $1.8 \AA$.

The two important features elucidated by this study are that (a) the size of the pendant groups can be adjusted to optimize the order regardless of the shape of the chromophore core, and that (b) the shape of the chromophore core controls the maximum achievable loading. From this preliminary study using theoretical "metamolecules" defined via our coarse-graining models, the optimal shape of the core should be linear.

A final application of multiscale theory is the effect of the slot (waveguide) width of a device on the overall EO coefficient. We consider the order that can be achieved in EO material under poling when the material is sandwiched between two electrodes. The poling field is applied between the two electrodes. Figure 6 shows the two (purple) plates separated along the $\mathrm{x}$-axis and the EO material in between them. The poling field is also parallel to the $\mathrm{x}$-axis. Periodic boundary conditions (as used for bulk materials) are assumed in the other two dimensions. 
The interaction of the chromophores with the walls of the slot include a Lennard-Jones, LJ, interaction potential, which is adapted from the ellipsoid-to-ellipsoid potential developed previously. The LJ potential is adapted from the contact function between the ellipsoid and the surface, setting the zero of the potential at the distance where the ellipsoid and the surface would be just touching. Additionally, the electrostatic interaction of the chromophores with the surface are calculated based on interactions with image charges induced in the surface by the charge distribution of the chromophores. Since the surface is conductive, any charge distribution is attracted to the image charges that it induces. In general, the strength of the interaction depends on the difference between the dielectric constants of the two materials. In this case, the EO material has a moderate dielectric constant ${ }^{41}$ (on the order of 4 to 20 ) while the conductor has a nearly infinite dielectric constant. Therefore the image charge is nearly equal in magnitude to that of the reflected object and the coulombic interaction is always attractive. The effect on ordering due to the presence of the walls is shown in Figure 6. Ordering converges to the bulk limit above an electrode spacing of approximately $50 \mathrm{~nm}$. However, below that limit, chromophore order decreases with slot (waveguide) width, which reduces chromophore loading, and consequently, the predicted electro-optic coefficient. The density of the material does decrease slightly (around $15 \%$ decrease, see Figure 6) with decreasing width, due to the mismatch of morphologies around $5 \mathrm{~nm}$ in from the walls (see Figure 6). The centrosymmetric order parameter, $P_{2}=\frac{1}{2}\left[3\left\langle\cos ^{2} \theta\right\rangle-1\right]$, is negative when the slot width is less than $30 \mathrm{~nm}$. This indicates that most of the chromophores are lying on the gold surface in such a way that their long axes are perpendicular to the x-axis; e.g. that the chromophores are lying parallel to the electrode surface. A set of such chromophores near the walls can be seen in Figure 6. The effects of these surface chromophores extend into the bulk.

The modeling shown in Figure 6 considers only the net order and density and does not consider whether the predicted morphology at the interface affects current leak through or device conductance, which would lower the poling field within the EO layer and further degrade performance.

a)

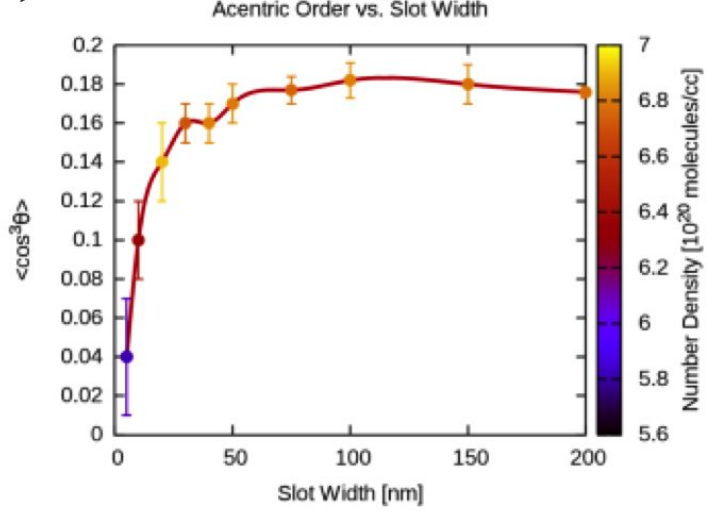

b)

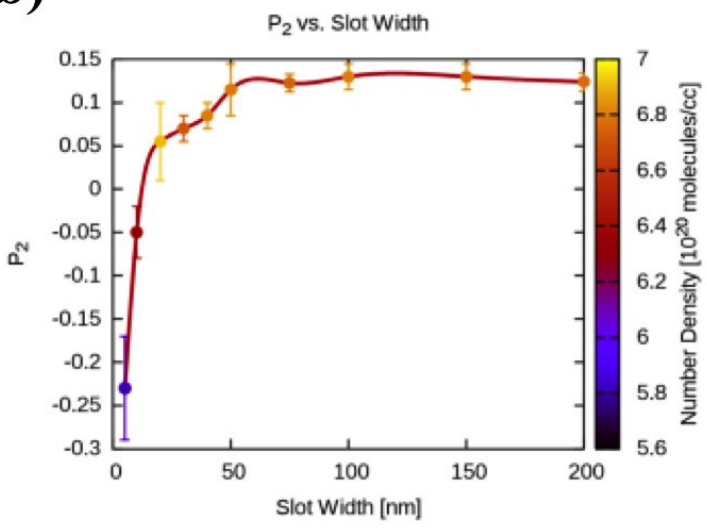

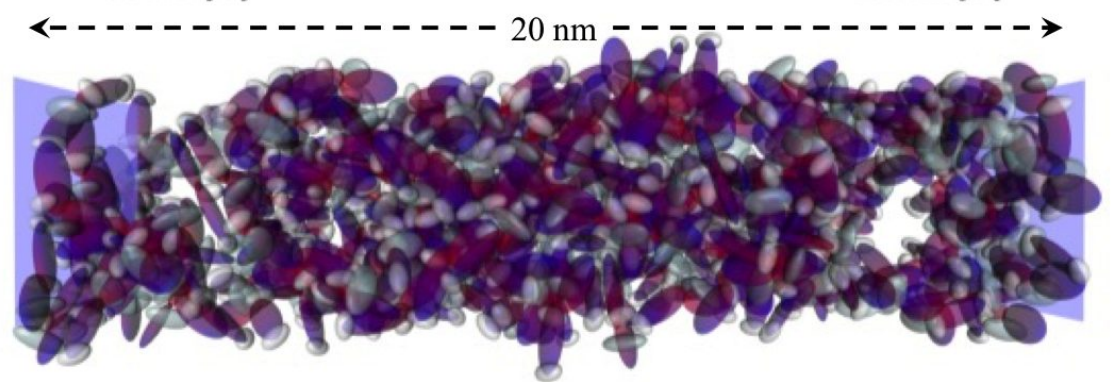

Figure 6 (a) Simulated acentric order is shown as a function of the plasmonic slot width. The number density of the films is illustrated by the color of the data points. (b) Simulated centrosymmetric order, $P_{2}=\left(3\left\langle\cos ^{2} \theta\right\rangle-1\right) / 2$, in the poling direction as a function of slot width. A snapshot from a simulation using a $20 \mathrm{~nm}$ slot is also shown 


\section{CONCLUSIONS}

The synergy between innovative device architectures and theoretical and computational models, that have been validated against existing chromophores and devices, has opened up possibilities for designing novel chromophores to meet the requirements for $\mathrm{SOH}$ and $\mathrm{POH}$ device architectures with respect to chipscale integration of electronics and photonics. Quantum mechanical (DFT) simulations predict trends in electro-optic activity for individual chromophores observed in both HRS and poling experiments. Large-scale, classical condensed-phase simulations of the morphology of EO chromophores near metallic surfaces explain the experimental observation that EO activity decreases as device waveguide widths become smaller. The modelling also suggests that some of the EO material may be acting as a surface passivation layer that causes background current leak-through to be somewhat attenuated. Such modelling methods may also be applied to simulating epitaxial growth from surfaces. These general principles of theory now admit evaluation of many different chromophores designs and processing methodologies.

We have used the AVA method to demonstrate that YLD124 should have an EO poling efficiency of around 3.0 $\mathrm{nm}^{2} / \mathrm{V}^{2}$, but poling experiments have observed an efficiency of about $1.5 \mathrm{~nm}^{2} / \mathrm{V}^{2}$. When the order is simulated directly from NPT ensemble simulations (without AVA conditioning) the predicted poling efficiency is about $1.5 \mathrm{~nm}^{2} / \mathrm{V}^{2}$. When minor modifications are made experimentally to pendant groups, without affecting the core structure, such materials do show the predicted poling efficiency of $3 \mathrm{~nm}^{2} / \mathrm{V}^{2}$. This suggests that the polinginduced order for some chromophore structures may be kinetically limited. ${ }^{10}$ YLD124 in 25\% PMMA has a poling efficiency of $1.0 \mathrm{~nm}^{2} / \mathrm{V}^{2}$. If poling-induced order were to remain constant, this would project a maximum poling efficiency of $4 \mathrm{~nm}^{2} / \mathrm{V}^{2}$ for a neat material, though this has not been achieved in practice. While earlier work using single-ellipsoid models had projected that order was heavily limited by intermolecular interactions, experience with different pendant groups and with YLD-124 doped into other chromophores has yielded poling efficiencies near this limit. Thus, the mobility of chromophore side groups and mobility of groups within the chromophore-containing matrix are likely crucial for obtaining satisfactory poling-induced order, and YLD-124 presents a poor matrix for itself.

Simulation software and protocol development has progressed to the point where multi-chromophore systems and interfacial layers can be simulated at an adequate scale to successfully predict properties. Future directions include determining how the interfacial morphology affects leak-through current. Furthermore, structures developed using molecular modelling (and the LoD methodology) may be used to define chromophore environments for ensembles of chromophores that can be used to simulate bulk optical properties. ${ }^{22}$

\section{Acknowledgement:}

We gratefully acknowledge the financial support of the National Science Foundation (DMR-1303080) and the Air Force Office of Scientific Research (FA9550-10-1-0558 and FA9550-15-1-0319). Any opinions, findings, and conclusions or recommendations expressed in this material are those of the author(s) and do not necessarily reflect the views of the National Science Foundation. Andreas F. Tillack has been partially supported by the U.S. Department of Energy, Office of Science, Office of Advanced Scientific Computing Research, Oak Ridge Leadership Computing facility under contract number DE-AC05-00OR22725.

\section{REFERENCES}

[1] Chen, A. and Murphy, E. J., [Broadband Optical Modulators: Science, Technology, and Applications], CRC Press, Boca Raton, FL, 1-548 (2012).

[2] Almeida, V. R., Xu, Q., Barrios, C. A. and Lipson, M., "Guiding and confining light in void nanostructure," Opt. Lett. 29(11), 1209-1211 (2004).

[3] Jalali, B., and Fathpour, S., “Silicon photonics,” J. Lightwave Technol. 24(12), 4600-4615 (2006). 
[4] Dong, P., Chen, Y. K., Duan, G. H., and Neilson, D. T., "Silicon photonic devices and integrated circuits," Nanophotonics 3(4-5), 215-228 (2014).

[5] Bowers, J. E., Komljenovic, T., Davenport, M., Hulme, J., Liu, A. Y., Santis, C. T., Spott, A., Srinivasan, S., Stanton, E. J., and Zhang, C., "Recent advances in silicon photonic integrated circuits," Proc. SPIE 9774, 977402-977402-18 (2016).

[6] Thomson, D., Zilkie, A., Bowers, J. E., Komljenovic, T., Reed, G. T., Vivien, L., Marris-Morini, D., Cassan, E., Virot, L., Fedeli, J. M., Hartmann, J. M., Schmid, J. H., Xu, D. X., Boeuf, F., O’Brien, P., Mashanovich, G. Z. and Nedeljkovic, M., "Roadmap on silicon photonics,” J. Opt. 18(7), 073003 (2016).

[7] Ozbay, E., "Plasmonics: Merging photonics and electronics at nanoscale dimensions," Science 311(5758), 189-193 (2006).

[8] Krasavin, A. V. and Zayats, A. V., "Active nanophotonic circuitry based on dielectric-loaded plasmonic waveguides,” Adv. Opt. Mater. 3(12), 1662-1690 (2015).

[9] Kinsey, N., Ferrera, M., Shalaev, V. M. and Boltasseva, A., "Examining nanophotonics for integrated hybrid systems: A review of plasmonic interconnects and modulators using traditional and alternative materials," J. Opt. Soc. Am. B 32(1), 121-142 (2015).

[10] Heni, W., Kutuvantavida, Y., Haffner, C., Zwickel, H., Kieninger, C., Wolf, S., Lauermann, M., Tillack, A., Johnson, L. E., Elder, D. L., Robinson, B. H., Freude, W., Koos, C., Leuthold, J. and Dalton, L. R., "Silicon-organic and plasmonic-organic photonics," ACS Photonics, ASAP article, doi: 10.1021/acsphotonics.7b00224 (2017) and references contained therein.

[11] Dalton, L. R., Benight, S. J., Johnson, L. E., Knorr, D. B., Kosilkin, L., Eichinger, B. E., Robinson, B. H., Jen, A. K. Y. and Overney, R. M., "Systematic nano-engineering of soft matter organic electrooptic materials," Chem. Mater. 23, 430-445 (2011).

[12] Johnson, L. E., Dalton, L. R. and Robinson, B. H., “Optimizing calculations of electronic excitations and relative hyperpolarizabilities of electrooptic chromophores,” Acc. Chem. Res. 47, 3258-3265 (2014).

[13] Tillack, A. F., Johnson, L. E., Rawal, M. and Dalton, L. R., "Modeling chromophore order: A guide for improving EO performance,” Mat. Res. Soc. Proc. 1698, mrss14-1698-jj08-05 (2014).

[14] Robinson, B. H., Johnson, L. E. and Eichinger, B. E., "Relation of system dimensionality and order parameters," J. Phys. Chem. B 119(7), 3205-3212 (2015).

[16] Dalton, L. R., Gunter, P., Jazbinsek, M., Kwon, O.-P. and Sullivan, P. A., [Organic Electro-Optics and Photonics: Molecules, Polymers, and Crystals], Cambridge University Press, Cambridge, UK, 1-293 (2015).

[17] Tillack, A. F. and Robinson, B. H., "A simple model for the benzene/hexafluorobenzene interaction," J. Phys. Chem. B. 121 (25), 6184-6188 (2017).

[18] Bale, D. H., Eichinger, B. E., Liang, W., Li, X., Dalton, L. R., Robinson, B. H., and Reid, P. J., "Dielectric dependence of the first molecular hyperpolarizability for electro-optic chromophores," J. Phys. Chem. B, 115(13), 3505-3513 (2011).

[19] Suponitsky, K. Y, Liao, Y. \& Masunov, A. E., "Electronic hyperpolarizabilities for donor-acceptor 
molecules with long conjugated bridges: Calculations versus experiment," J. Phys. Chem., A. 113, 10994-11001 (2009).

[20] Sears, J.S., Coropceanu, V. \& Bredas, J. L., "Understanding the density functional dependence of calculated electronic couplings in organic semiconductors," J. Phys. Chem. Lett., 4, 919-924 (2013).

[21] Frisch, M. J.; Trucks, G. W.; Schlegel, H. B.; Scuseria, G. E.; Robb, M. A.; Cheeseman, J. R.; Scalmani, G.; Barone, V.; Mennucci, B.; Petersson, G. A.; Nakatsuji, H.; Caricato, M.; Li, X.; Hratchian, H. P.; Izmaylov, A. F.; Bloino, J.; Zheng, G.; Sonnenberg, J. L.; Hada, M.; Ehara, M.; Toyota, K.; Fukuda, R.; Hasegawa, J.; Ishida, M.; Nakajima, T.; Honda, Y.; Kitao, O.; Nakai, H.; Vreven, T.; Montgomery Jr., J. A.; Peralta, J. E.; Ogliaro, F.; Bearpark, M. J.; Heyd, J.; Brothers, E. N.; Kudin, K. N.; Staroverov, V. N.; Kobayashi, R.;

Normand, J.; Raghavachari, K.; Rendell, A. P.; Burant, J. C.; Iyengar, S. S.; Tomasi, J.; Cossi, M.; Rega, N.; Millam, N. J.; Klene, M.; Knox, J. E.; Cross, J. B.; Bakken, V.; Adamo, C.; Jaramillo, J.; Gomperts, R.; Stratmann, R. E.; Yazyev, O.; Austin, A. J.; Cammi, R.; Pomelli, C.; Ochterski, J. W.; Martin, R. L.; Morokuma, K.; Zakrzewski, V. G.; Voth, G. A.; Salvador, P.; Dannenberg, J. J.; Dapprich, S.; Daniels, A. D.; Farkas, Ö.; Foresman, J. B.; Ortiz, J. V.; Cioslowski, J.; Fox, D. J. Gaussian 09, Revision E.01; Gaussian, Inc.: Wallingford, CT, USA, 2009.

[22] Isborn, C. M. Excited States in the Condensed Phase, in [TACC 2016], Seattle (2016) 